\title{
Data Analysis - Transfer learning for non-image data in clinical research: a scoping review
}

\author{
Andreas Ebbehoj, Mette Thunbo, Ole Emil Andersen, Michala Vilstrup Glindtvad, Adam Hulman \\ Disclaimer: the calculations and numbers are in the focus of this document, and therefore, the text has not \\ been edited at the same level of detail as the corresponding manuscript. \\ sessionInfo() \\ \#\# R version 4.1.0 (2021-05-18) \\ \#\# Platform: x86_64-apple-darwin17.0 (64-bit) \\ \#\# Running under: macOS Big Sur 10.16 \\ \#\# \\ \#\# Matrix products: default \\ \#\# BLAS: /Library/Frameworks/R.framework/Versions/4.1/Resources/lib/libRblas.dylib \\ \#\# LAPACK: /Library/Frameworks/R.framework/Versions/4.1/Resources/lib/libRlapack.dylib \\ \#\# \\ \#\# locale: \\ \#\# [1] en_US.UTF-8/en_US.UTF-8/en_US.UTF-8/C/en_US.UTF-8/en_US.UTF-8 \\ \#\# \\ \#\# attached base packages: \\ \#\# [1] stats graphics grDevices utils datasets methods base \\ \#\# \\ \#\# other attached packages: \\ \#\# [1] riverplot_0.10 zoo_1.8-9 purrr_0.3.4 stringr_1.4.0 readxl_1.3.1 \\ \#\# \\ \#\# loaded via a namespace (and not attached):

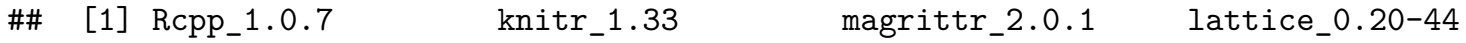 \\ \#\# [5] rlang_0.4.11 fansi_0.5.0 tools_4.1.0 grid_4.1.0 \\ \#\# [9] xfun_0.24 utf8_1.2.2 htmltools_0.5.1.1 ellipsis_0.3.2 \\ \#\# [13] yaml_2.2.1 digest_0.6.27 tibble_3. 1.3 lifecycle_1.0.0 \\ \#\# [17] crayon_1.4.1 RColorBrewer_1.1-2 vctrs_0.3.8 evaluate_0.14 \\ \#\# [21] rmarkdown_2.9 \\ \#\# [25] cellranger_1.1.0 \\ stringi_1.7.3 compiler_4.1.0 \\ pillar_1.6.2
}

The document was compiled at 2021-12-10 11:37:29.

\section{Research questions defined in the scoping review protocol}

The results are discussed in a different order in the manuscript. 


\section{To what extent is transfer learning (TL) used in clinical research?}

n_articles <- nrow(data)

n_articles

\#\# [1] 83

We have identified 83 articles.

\section{What is transfer learning used for? (e.g. improve predictions or make them feasible)}

The studies' aims have been extracted from a clinical point of view. The frequencies of some common terms are shown below. Note that the aims have been written by the review team and therefore might reflect their word use preferences, although we aimed to be consistent with the specific terms used in the given articles.

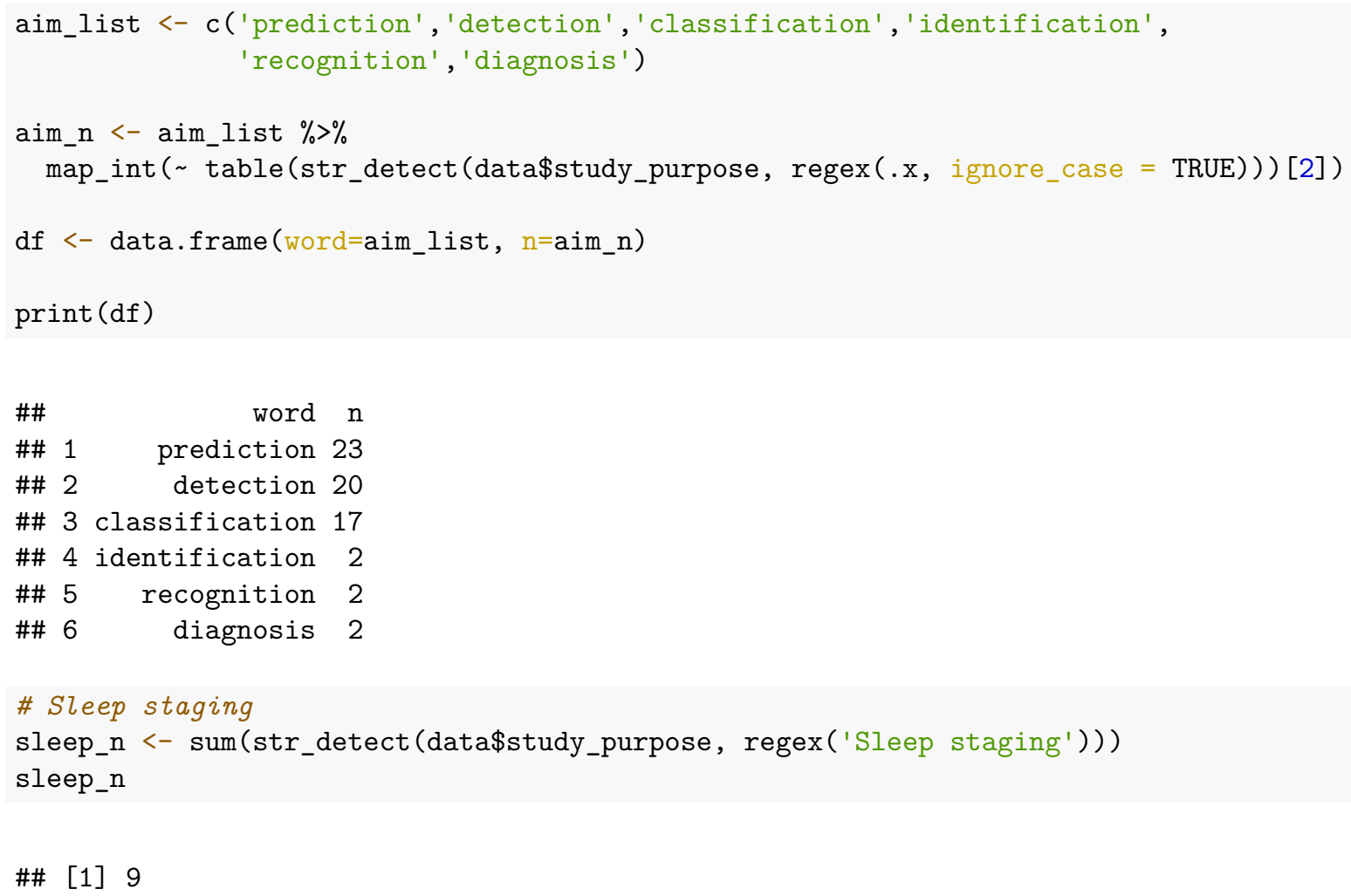

The most common specific aim was 'sleep staging' with 9 articles.

The complete list of study aims are shown below.

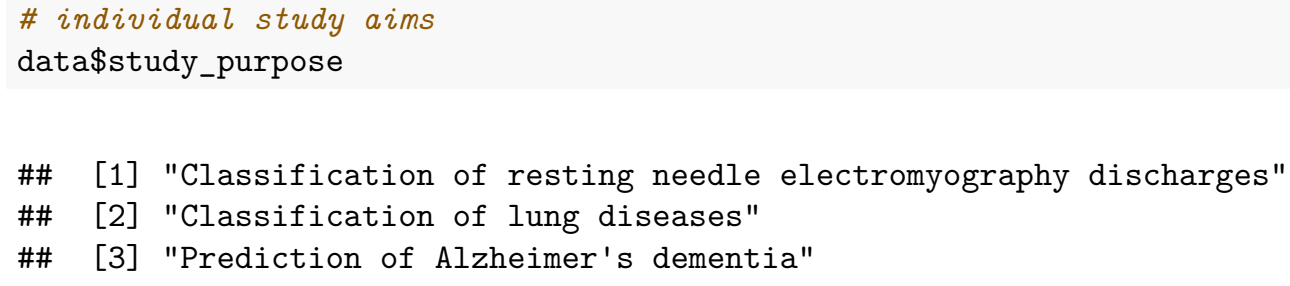




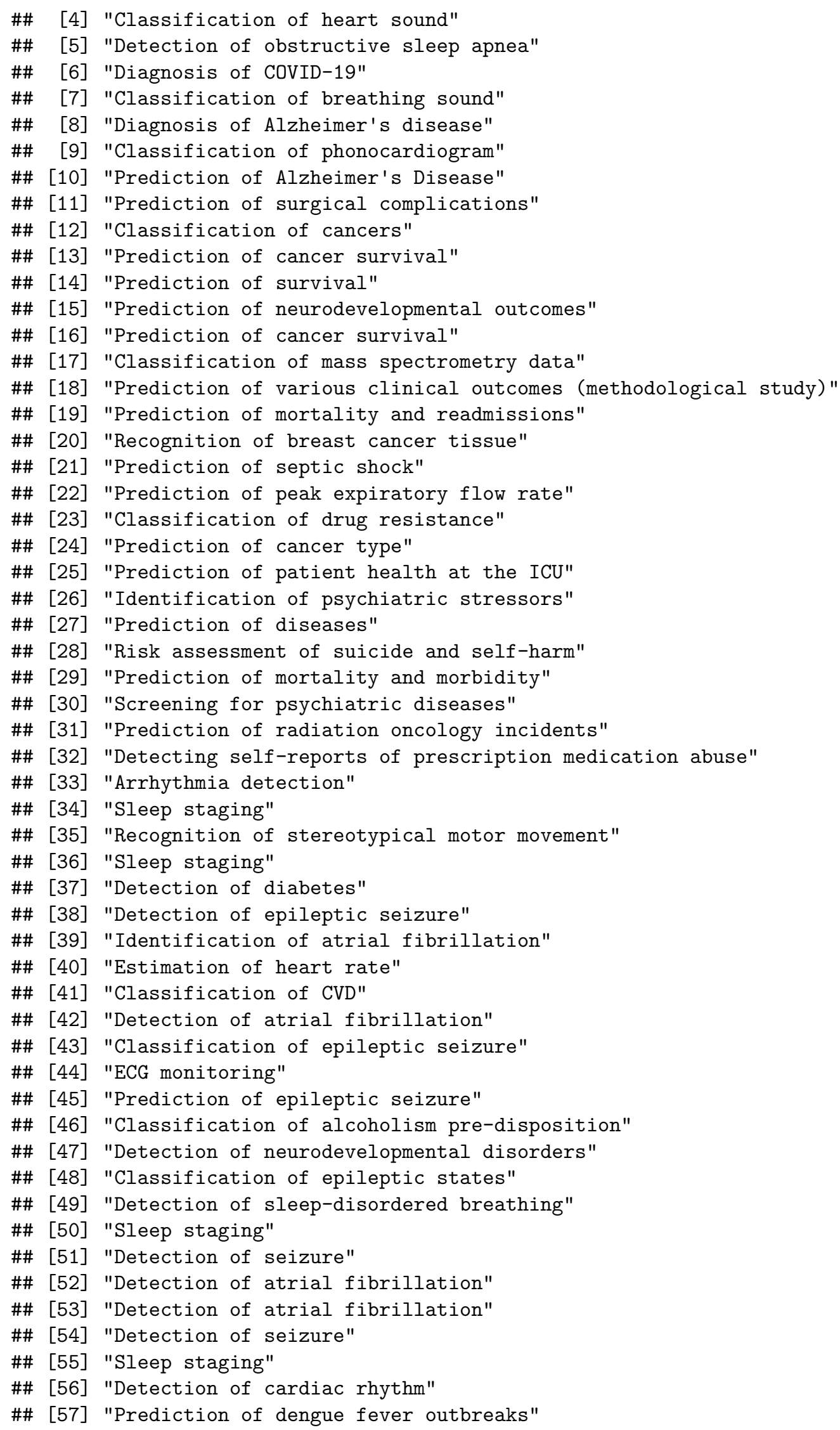




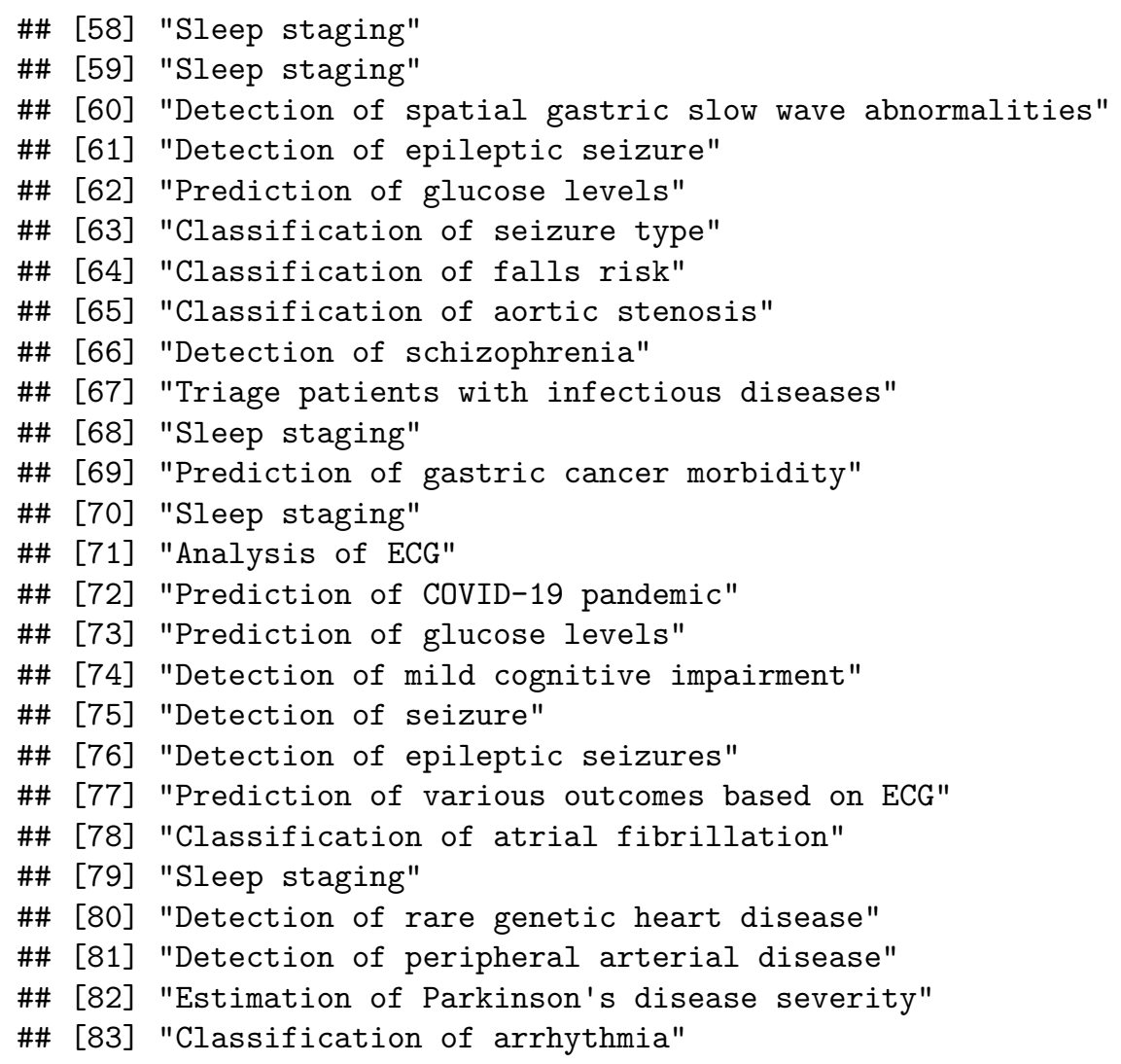

It is more difficult to summarize (at least quantitatively) the specific reason of using TL. As 4 out of 5 studies compared the performance of the TL model to a non-TL solution, performance improvement seems to be the \#1 reason. Also, we observed many examples where the target dataset were very small (sometimes subsets of the source data), limiting the utility of ML methods without the use of transfer learning. In the most extreme cases, the target dataset included data from a specific individual, aiming to develop individualized models, but still utilising data from the whole study population. Similarly, instead of zooming into individuals, other studies utilised data from a disease group (e.g. cancer) to zoom into a specific one in that group (e.g. lung cancer).

\section{In which areas of clinical research is transfer learning used?}

Medical field was assigned by the reviewers during data extraction. Often more fields were noted in the first place and then the best matching one was chosen in consensus by the reviewers.

sort(table(data\$study_field), decreasing $=\mathrm{T}$ )

$\begin{array}{rrr}\# \# & & \\ \# \# & \text { Neurology } & \text { Cardiology } \\ \# \# & 26 & 18 \\ \text { \#\# } & \text { Psychiatry } & \text { Endocrinology } \\ \text { \#\# } & 5 & 3 \\ \# \# & \text { Pulmonology } & \text { Intensive care } \\ \# \# & 3 & \\ \text { \#\# } & \text { Pharmacology } & \text { Gastroenterology } \\ \text { \#\# } & 2 & 1\end{array}$

$\begin{array}{rr}\text { Genetics } & \text { Infectious diseases } \\ 5 & 5 \\ \text { Epidemiology } & \text { Pathology } \\ 3 & 3 \\ \text { Neonatology } & \text { Otorhinolaryngology } \\ 2 & 2 \\ \text { Geriatrics } & \text { Oncology } \\ 1 & 1\end{array}$




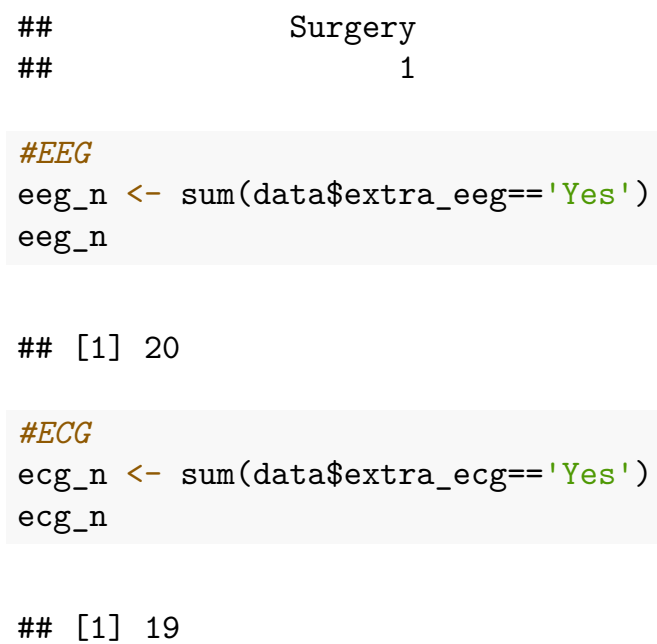

Two types of studies stood out with very high frequencies: analysis of EEG $(n=20)$ and ECG $(n=19)$ data.

\section{Who uses transfer learning? (computer scientists/clinicians/together)}

The authors affiliations were considered in this analysis as a proxy for their professional background, as this information is available in all articles. Technical affiliations included institutions in computer science, engineering, mathematics or similar, while clinical affiliations were defined by ones from medicine, health or similar. Frequencies are shown below.

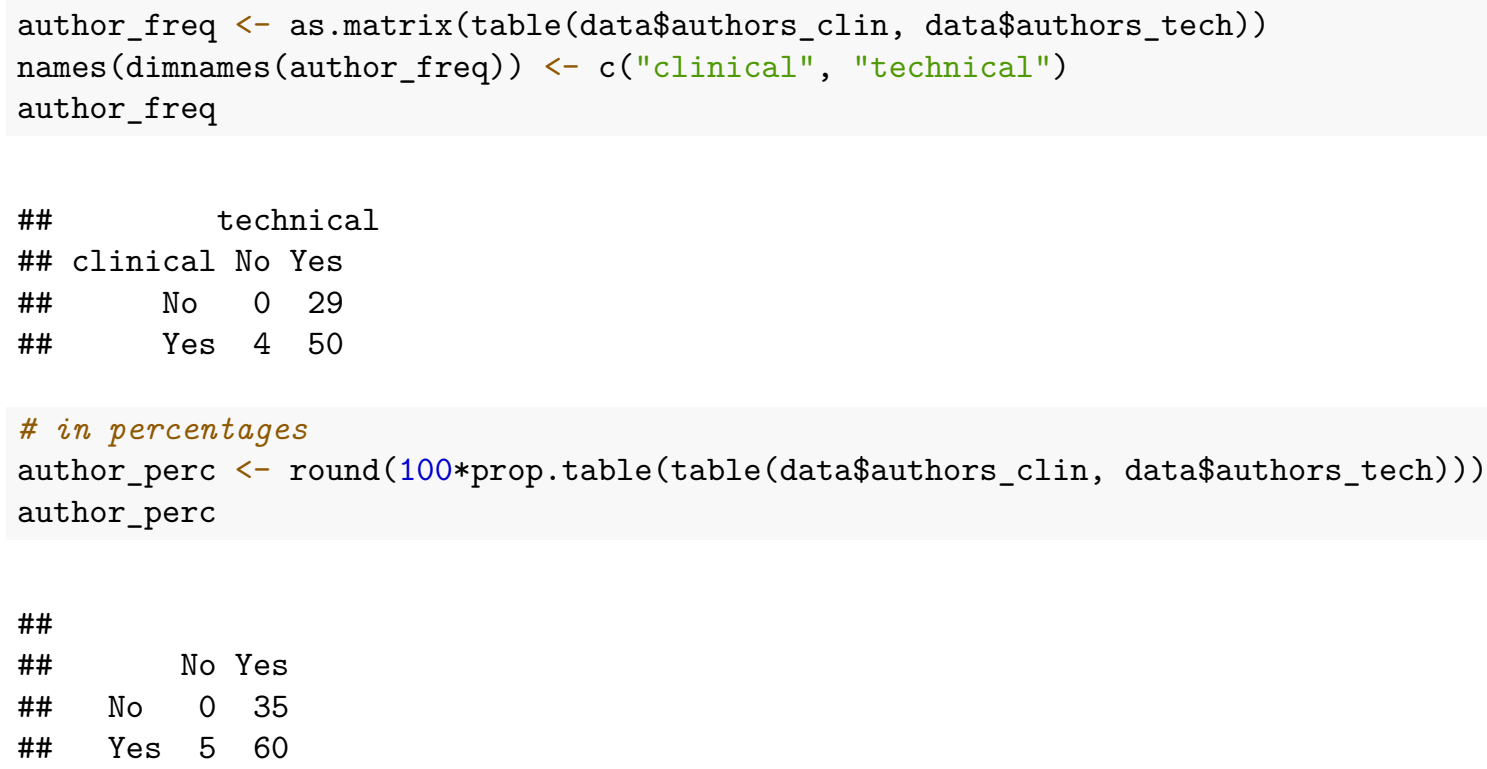

Three out of five studies (60\%) were conducted in collaboration between authors from both clinical and technical backgrounds. Studies conducted by authors only from a technical background $(35 \%)$ were more common than those from only a clinical background $(5 \%)$.

\section{Where are the results published? (clinical/interdisciplinary journals)}




\begin{tabular}{|c|c|}
\hline \multicolumn{2}{|l|}{ \#\# } \\
\hline \#\# & Annu Int Conf IEEE Eng Med Biol Soc \\
\hline \#\# & $\begin{array}{ll}9 & 9\end{array}$ \\
\hline \#\# & IEEE J Biomed Health Inform \\
\hline \#\# & 4 \\
\hline \#\# & Physiol Meas \\
\hline \#\# & 4 \\
\hline \#\# & Sci Rep \\
\hline \#\# & 4 \\
\hline \#\# & BMC Med Inform Decis Mak \\
\hline \#\# & 3 \\
\hline \#\# & Nat Commun \\
\hline \#\# & 3 \\
\hline \#\# & Comput Biol Med \\
\hline \#\# & 2 \\
\hline \#\# & Comput Methods Programs Biomed \\
\hline \#\# & 2 \\
\hline \#\# & PLoS One \\
\hline \#\# & 2 \\
\hline \#\# & Sleep \\
\hline \#\# & 2 \\
\hline \#\# & AMIA Jt Summits Transl Sci Proc \\
\hline \#\# & 1 \\
\hline \#\# & Ann Emerg Med \\
\hline \#\# & 1 \\
\hline \#\# & Biocybernetics and Biomedical Engineering \\
\hline \#\# & 1 \\
\hline \#\# & Bioinformatics \\
\hline \#\# & 1 \\
\hline \#\# & Biomed Eng Online \\
\hline \#\# & 1 \\
\hline \#\# & Biomedical Physics and Engineering Express \\
\hline \#\# & $\begin{array}{lllll}21 & 1\end{array}$ \\
\hline \#\# & Biomedical Signal Processing and Control \\
\hline \#\# & $\begin{array}{lll}-1 & 1\end{array}$ \\
\hline \#\# & Biosensors (Basel) \\
\hline \#\# & 1 \\
\hline \#\# & BMC Bioinformatics \\
\hline \#\# & 1 \\
\hline \#\# & Clin Neurophysiol \\
\hline \#\# & 1 \\
\hline \#\# & Comput Intell Neurosci \\
\hline \#\# & $\begin{array}{ll}1 \\
1\end{array}$ \\
\hline \#\# & Comput Math Methods Med \\
\hline \#\# & 1 \\
\hline \#\# & Diabetes Technol Ther \\
\hline \#\# & 1 \\
\hline \#\# & Eur Neurol \\
\hline \#\# & 1 \\
\hline \#\# & Front Aging Neurosci \\
\hline \#\# & 1 \\
\hline
\end{tabular}


Front Digit Health

Front Hum Neurosci

Front Neurol

1

Front Psychiatry

1

Front Psychol

1

Health Inf Sci Syst

1

1

Healthcare (Basel)

IEEE Trans Biomed Circuits Syst

IEEE Trans Biomed Eng

IEEE Trans Neural Netw Learn Syst

IEEE Transactions on Neural Systems and Rehabilitation Engineering

Inform Med Unlocked

1

1

Int J Comput Assist Radiol Surg

Int J Environ Res Public Health

1

Int J Neural Syst

1

IRBM

1

$\mathrm{J}$ Alzheimers Dis

J Biomed Inform

J Biomed Res

1

$\mathrm{J}$ Healthc Inform Res

1

$\mathrm{J}$ Med Internet Res

1

$\mathrm{J}$ Neural Eng

J Neurosci Methods

\section{1}

Journal of Medical and Biological Engineering

Journal of Medical Imaging and Health Informatics 


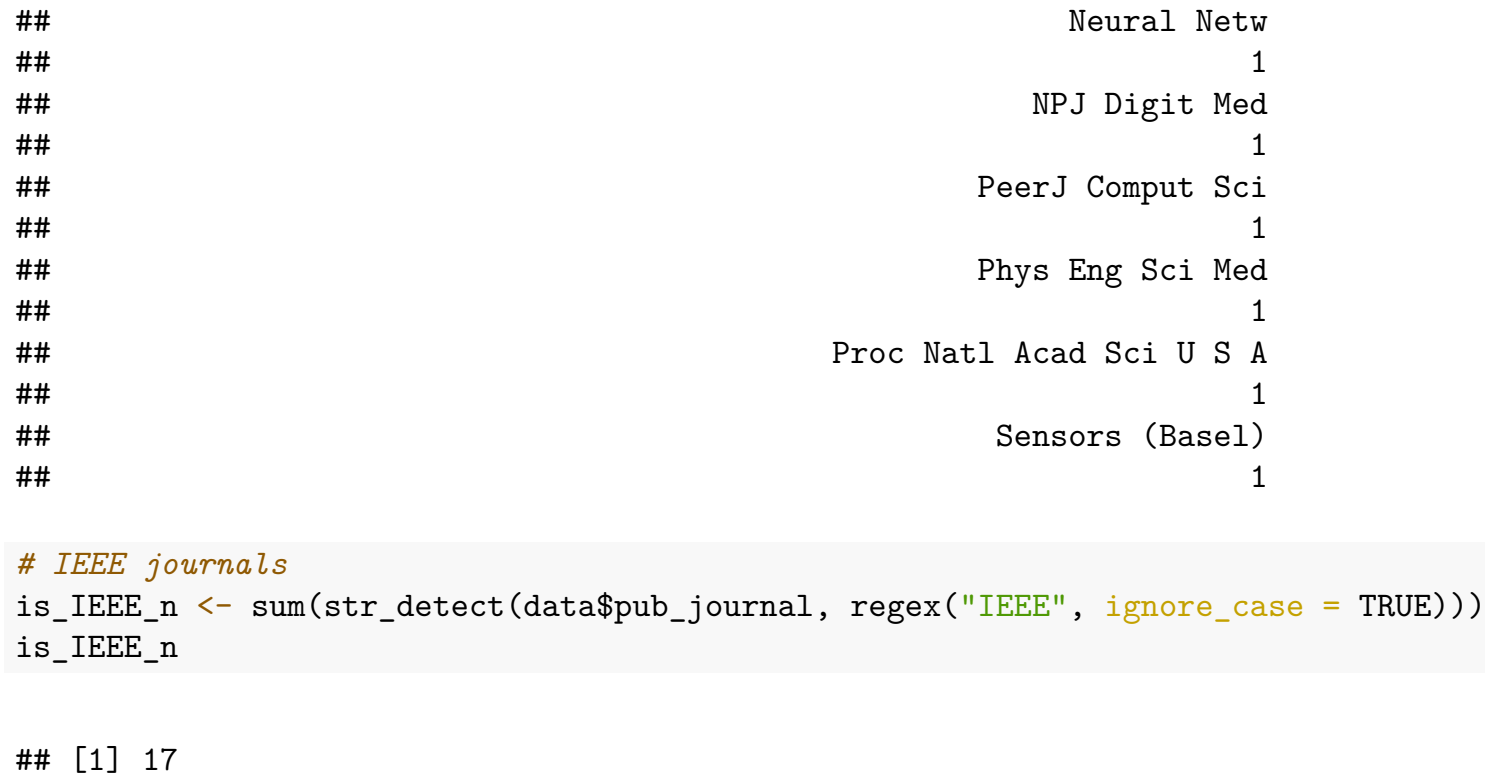

The overall picture is that the majority of articles were published in interdisciplinary journals with a significant technical focus, despite that we considered only medical databases (PubMed, EMBASE, CINAHL). One in five articles $(n=17)$ were published in IEEE-related journals (or proceedings). There are almost no clinical mainstream journals in the list, but a few general scientific journals (Sci Rep, Nat Commun, Plos One) with a broader audience.

\section{What type of non-image data is transfer learning applied for? (e.g. tabular, time series, text, voice)}

type_freq <- table(data\$data_type_target_domain)

type_freq

\section{\#\#}

\#\# Audio Tabular Text Time series

$\begin{array}{llll}\text { \#\# } & 10 & 15 & 7\end{array}$

round (100*prop.table(type_freq))

\#\#

\#\# Audio Tabular $\quad$ Text Time series

$\begin{array}{lrrrr}\text { \#\# } & 12 & 18 & 8 & 61\end{array}$

\# different source and target types

type_diff_n $<-$ sum (data\$data_type_source_diff=='Yes' $)$

type_diff_n

\#\# [1] 36 


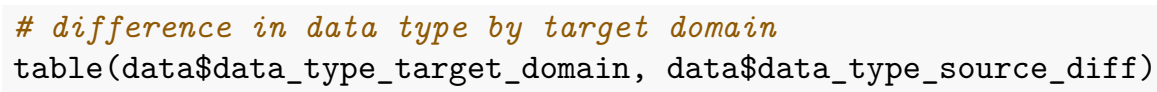

The most common target data type was time series $(n=51)$, then tabular data $(n=15)$, audio $(n=10)$ and text $(n=7)$.

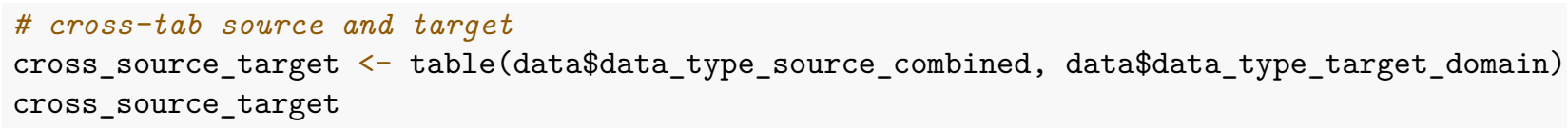

We were surprised seeing many studies $(n=36)$ reusing models from a different source data type than the target itself. The frequencies of different combinations are shown in the last table above, where the rows 
represent the source domain and the columns represent the target domain. Three studies included and compared source models of different types, that's why multiple types appear in some rows. These studies contributed with two records to the Sankey diagram.

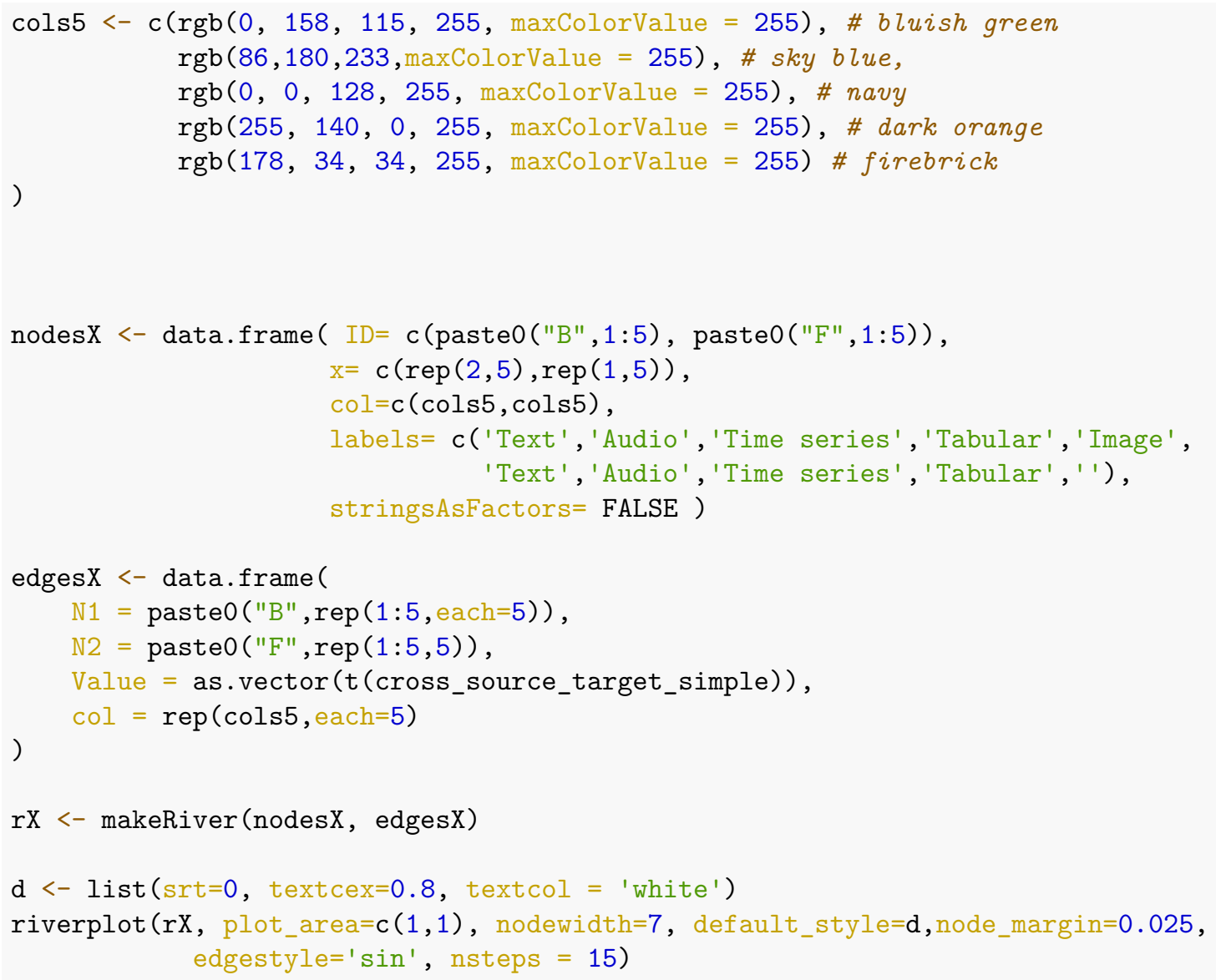




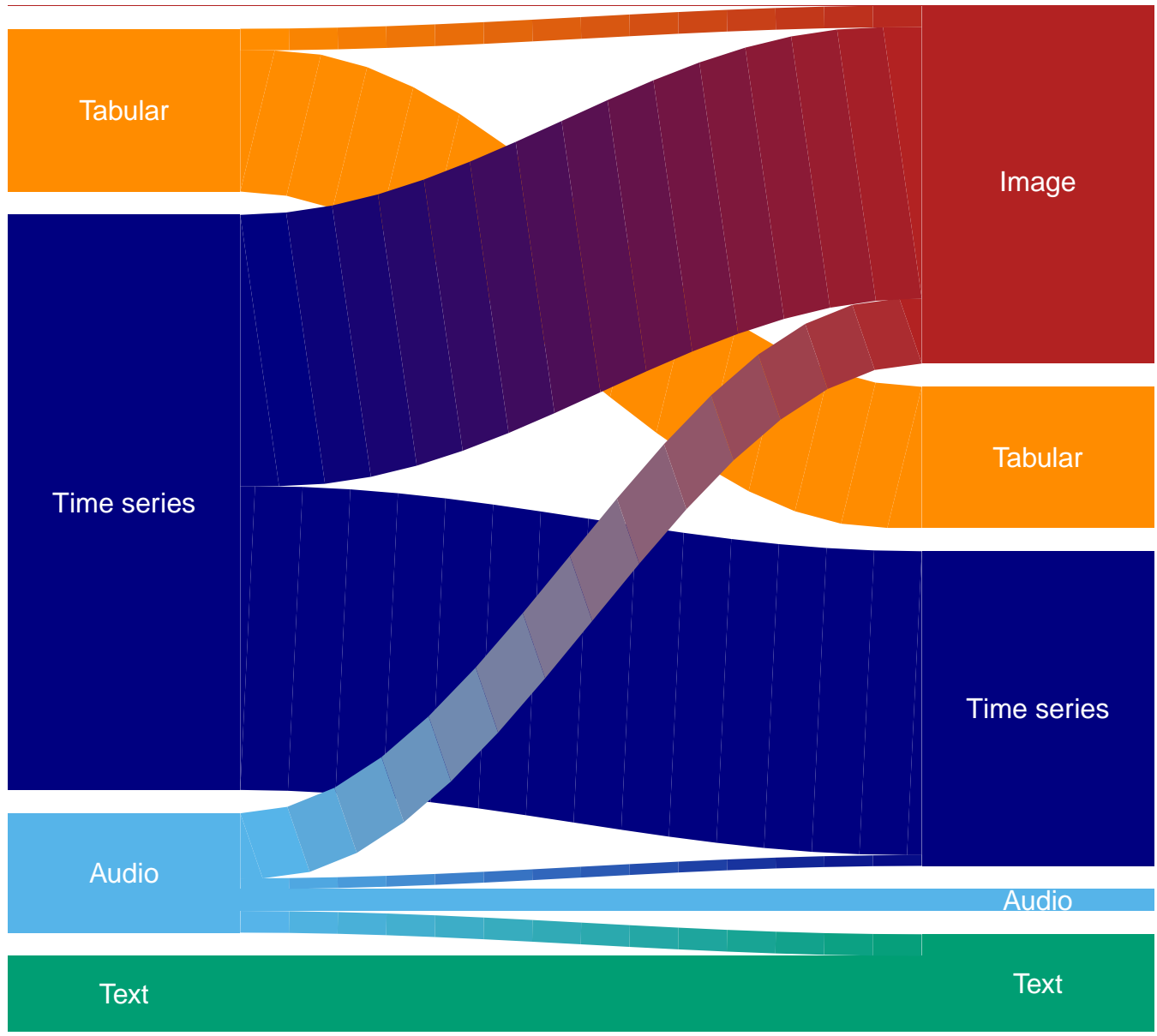

setEPS()

postscript ("Figure3.eps")

riverplot (rX, plot_area=c $(1,1)$, nodewidth=7, default_style=d, node_margin=0.025, edgestyle='sin', nsteps = 15)

$\operatorname{dev} \cdot \operatorname{off}()$

\#\# pdf

\#\# 2

The riverplot package has a well-documented issue (see p. 13 in the package documentation) i.e. displaying vertical white lines, that we tried to overcome by increasing the number of interpolating segments. This led to unwated triangles both on the left and the right sides, which are covered by rectangles of a matching color (done outside of $\mathrm{R}$ ) in the version used in the manuscript. 


\section{What are the sizes of the datasets used in transfer learning (both in the source and target domains)?}

Differences in units made it unfeasible to examine this question. One of the main contributing factors to this was that source and target data types were different more often than we expected $(n=36)$. E.g. audio recordings in minutes, number of pictures, tweets.

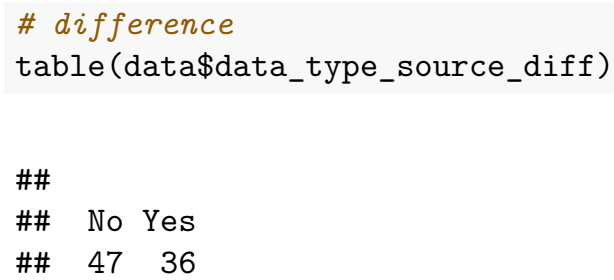

\section{What kind of models are reused with transfer learning? (e.g. CNN, LSTM)}

As expected, neural network-based methods dominated the type of reused models, with a very few exceptions $(n=3)$ reusing traditional statistical models or SVMs. The frequencies are shown below (the numbers don't necessarily add up to the number of articles, because some studies used multiple methods and/or their combinatation).

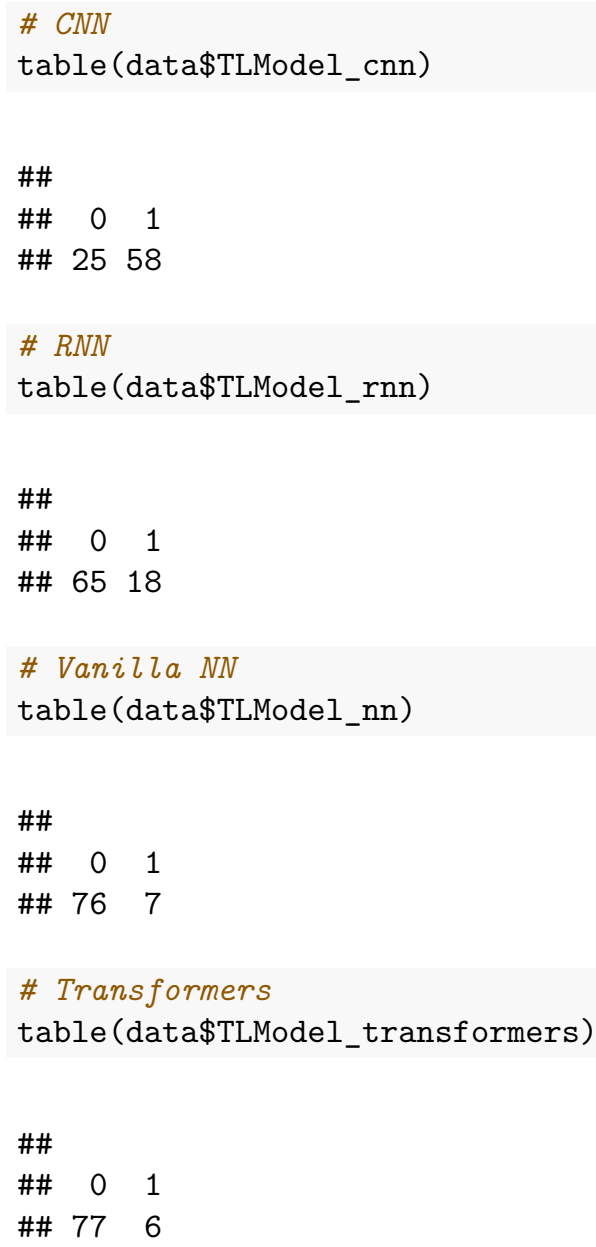




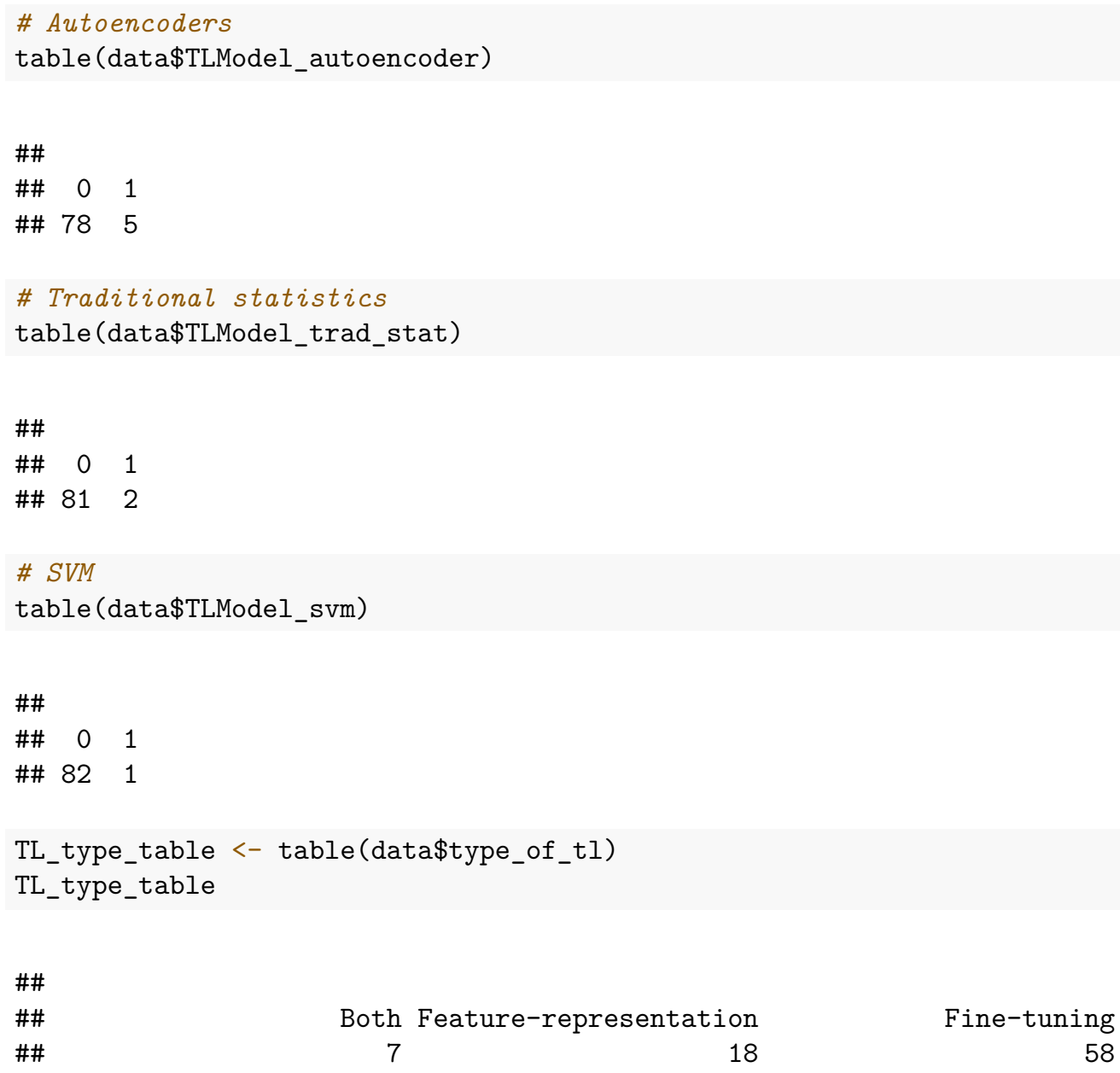

Fine-tuning was approx. $3 x$ as common $(n=58)$ as feature representation transfer $(n=18)$. Seven studies used both. 


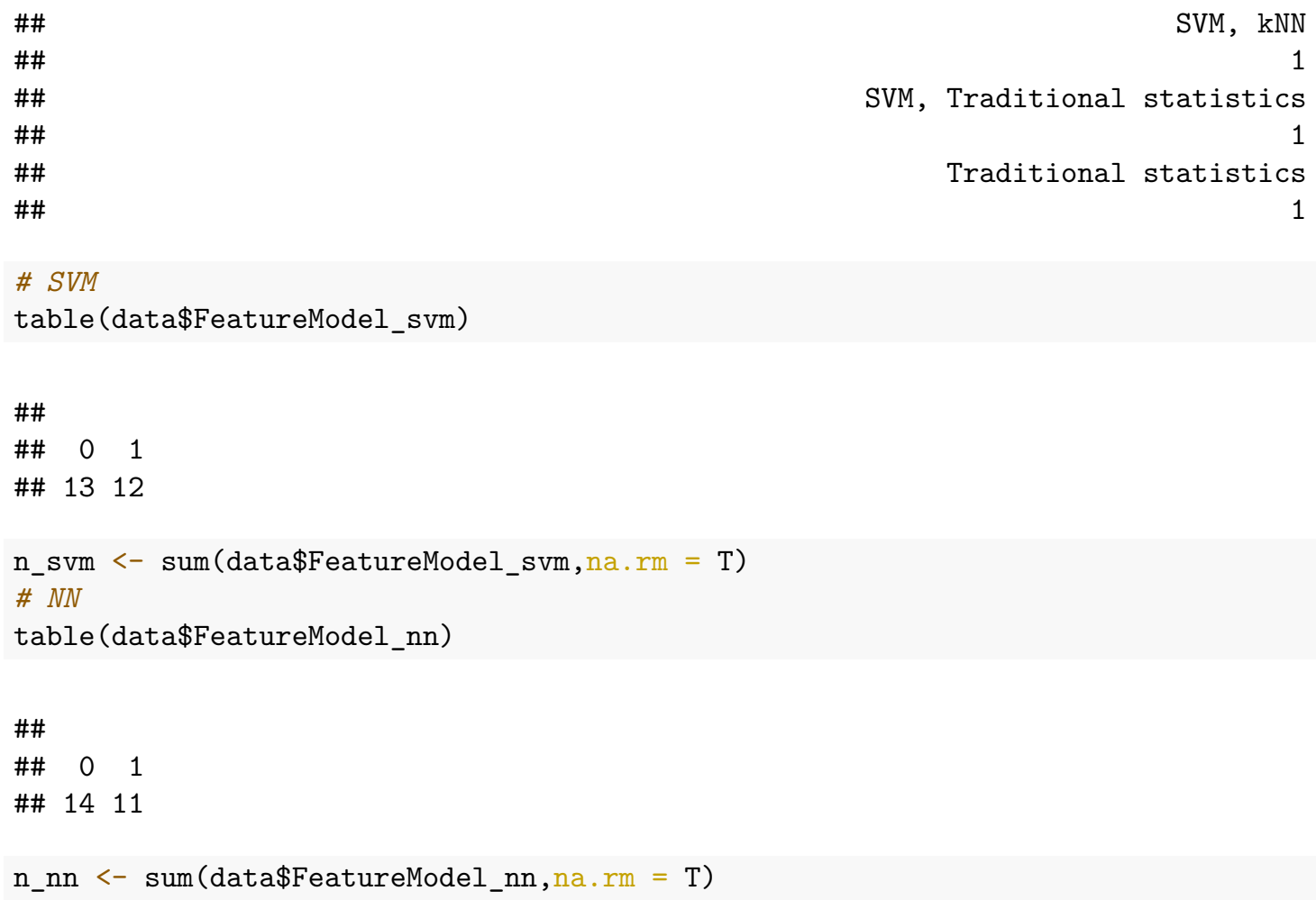

Support vector machine (SVM) was the most common final model $(n=12)$ out of the 25 studies using feature representation transfer, followed closely by 'vanilla' neural networks. Seven studies used and compared multiple models.

\section{Are the reused models and implementation code publicly available?}

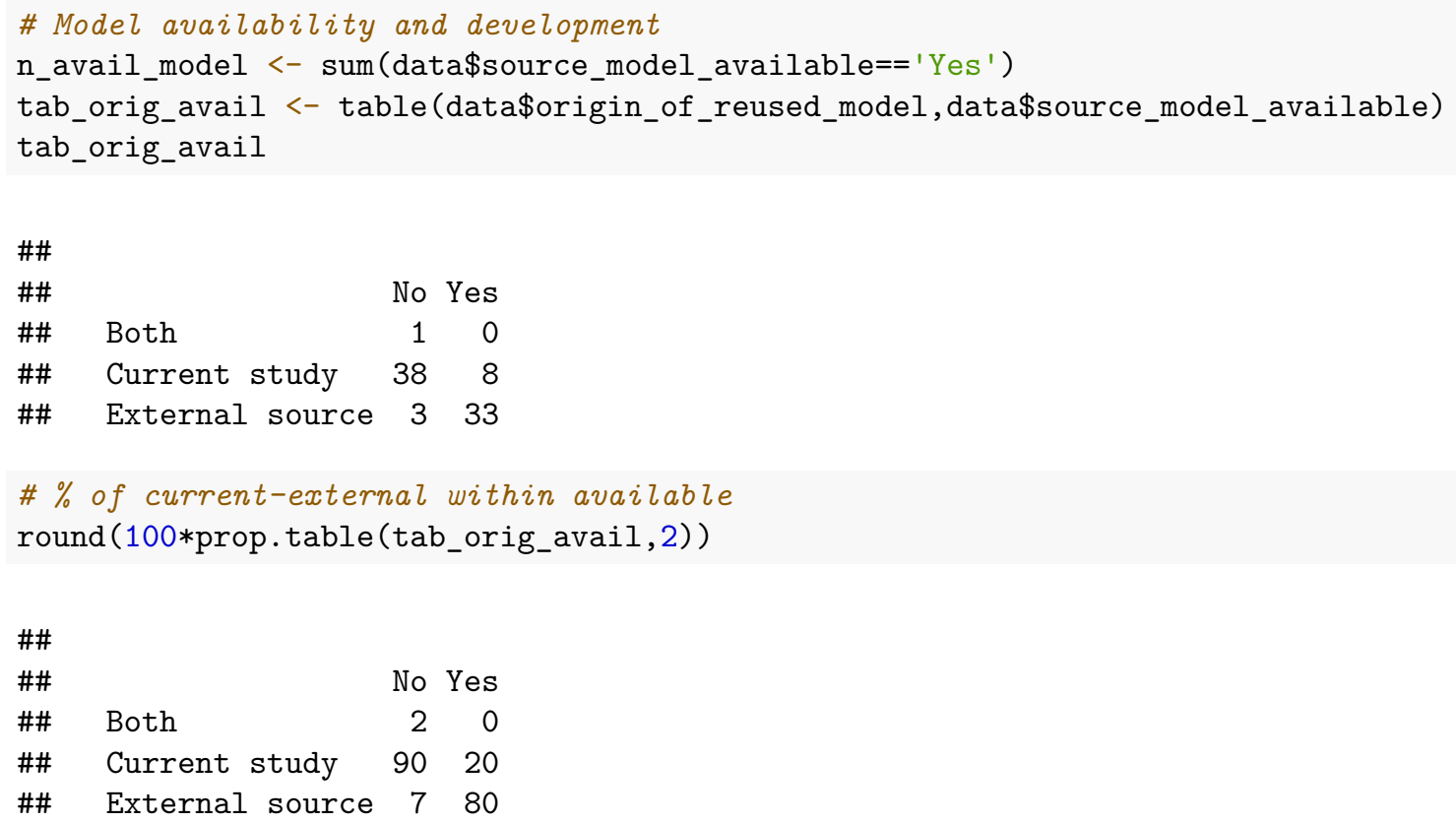




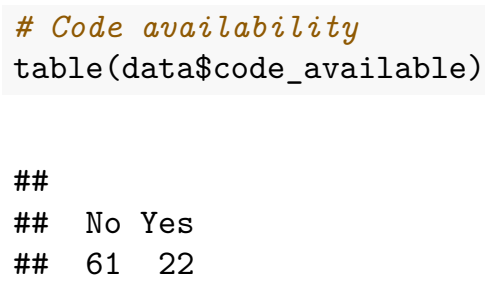

Half of the studies used an publicly available model $(n=41)$, among which those models dominated that were readily available in the used software packages.

Code availability was low, only one in four studies published their code, most of these were available on GitHub.

\section{What software was used to implement transfer learning? (e.g. Python, R, MATLAB)}

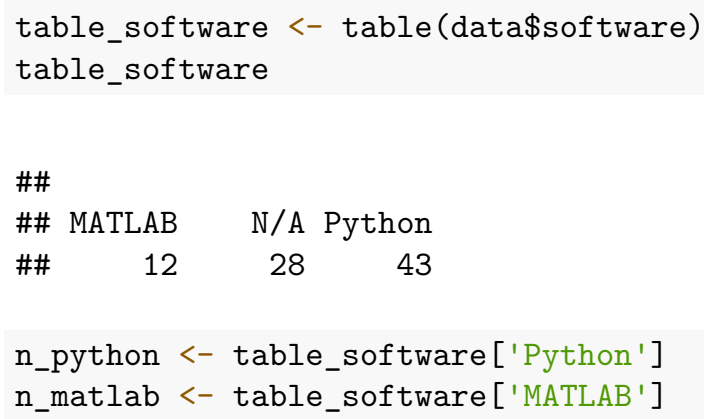

Python ( $n=43)$ was the dominant software among those who reported this detail, followed by MATLAB $(n=12)$. One in three articles did not report the software they used at all. We did not find a single study using R.

These numbers represent the software used for the analysis and not for data processing. However, if the authors reported software/packages used for data processing, but not for the analysis, then we assumed that they used the same. If packages like PyTorch, tensorflow or keras were mentioned, then we assumed that the authors used Python (their native language), as these packages became available in R only recently.

\section{Is the transfer learning solution compared to a (benchmark) solution without trans- fer learning? If yes, what are the costs or benefits of transfer learning?}

table (data\$tl_compared_to_non_tl)

\#\#

\#\# No Yes

\#\# $16 \quad 67$

Four in five studies compared the TL results to a non-TL solution. The majority of these studies included multiple models and performance metrics and therefore it is difficult to quantify the costs/benefits of using TL, however shorter running time/less iteration needed were mentioned several times among the benefits. 


\section{Trend in using transfer learning? (calendar year)}

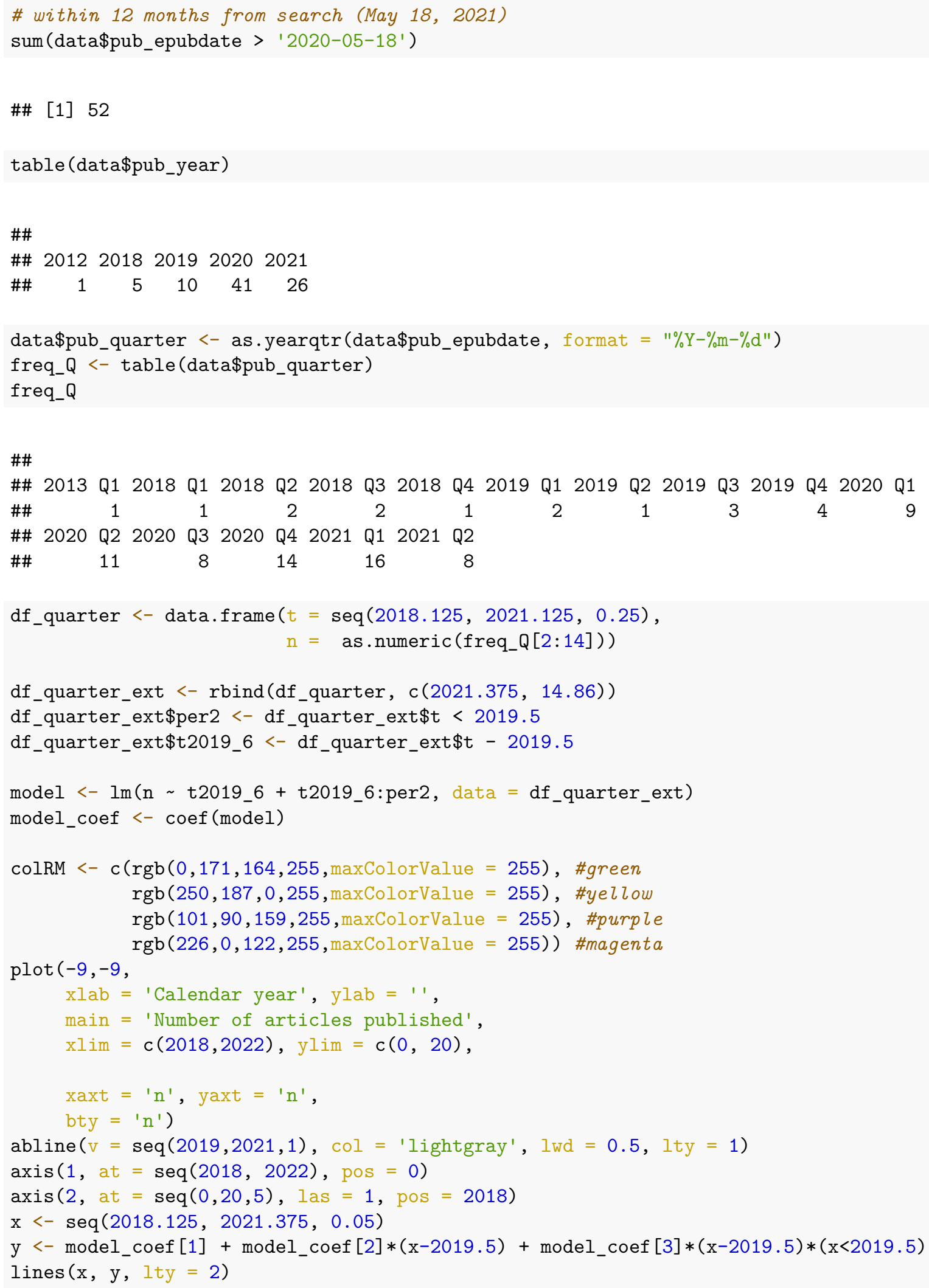




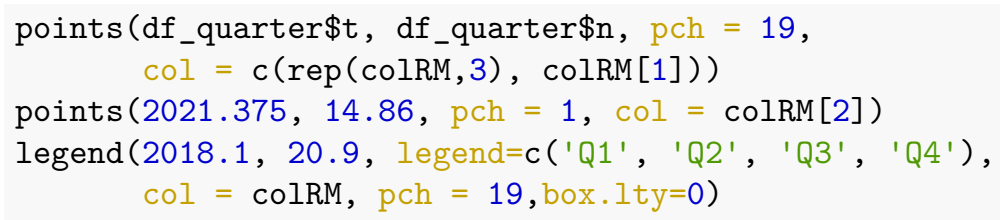

Number of articles published

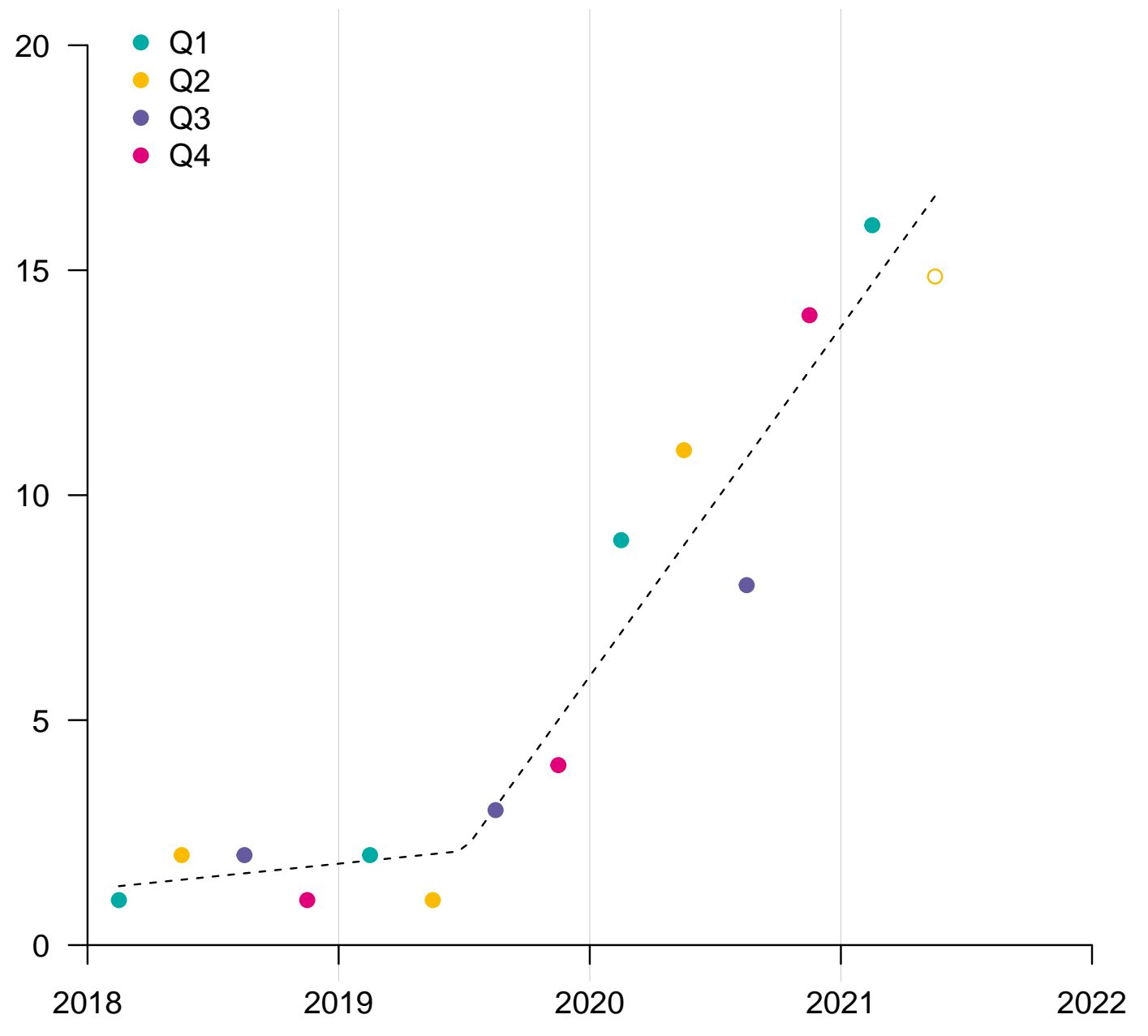

Calendar year

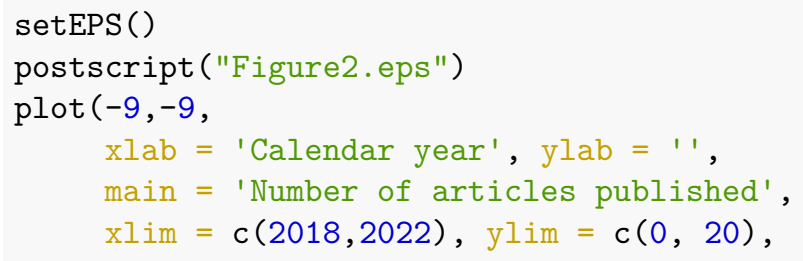




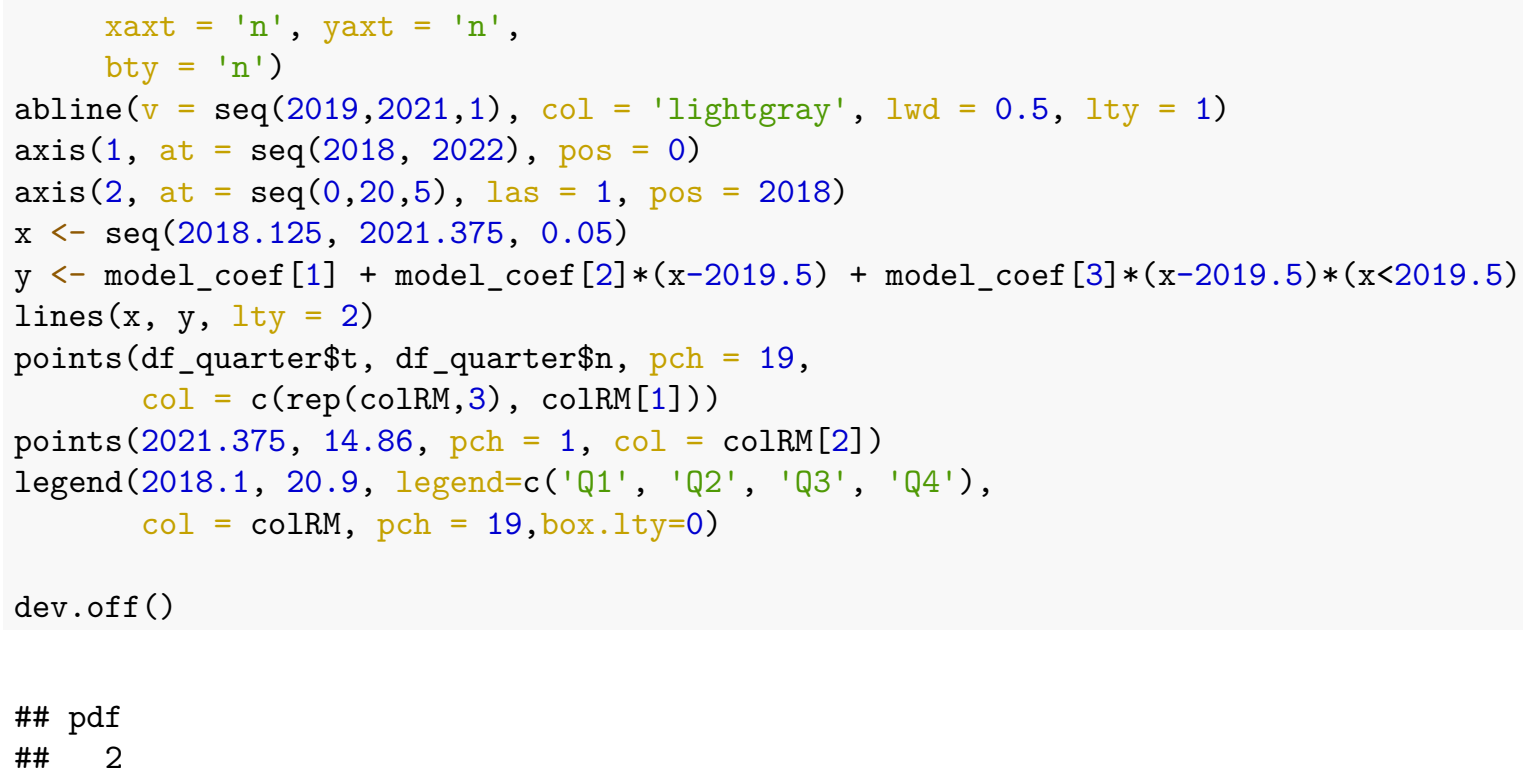

The number of articles seems to increase rapidly from 2019 , although the overall number is still quite low

\section{Questions formulated after publishing the protocol}

\section{To what extend are open access datasets used?}

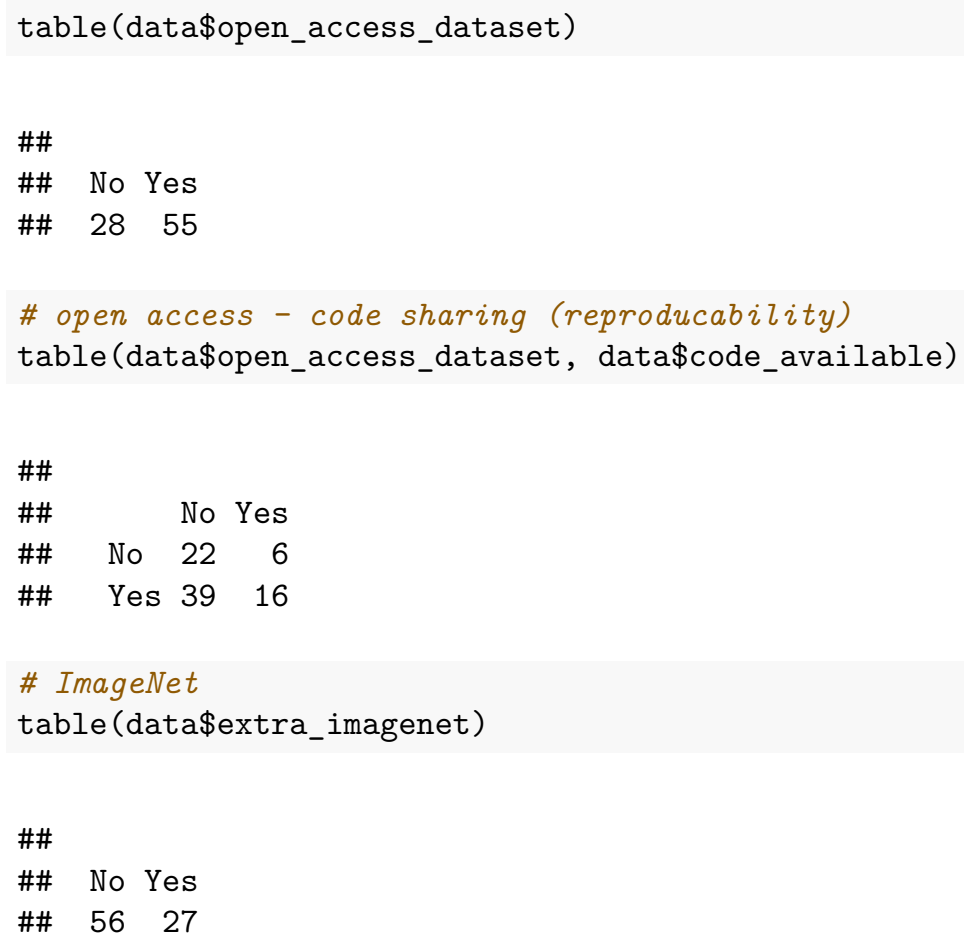




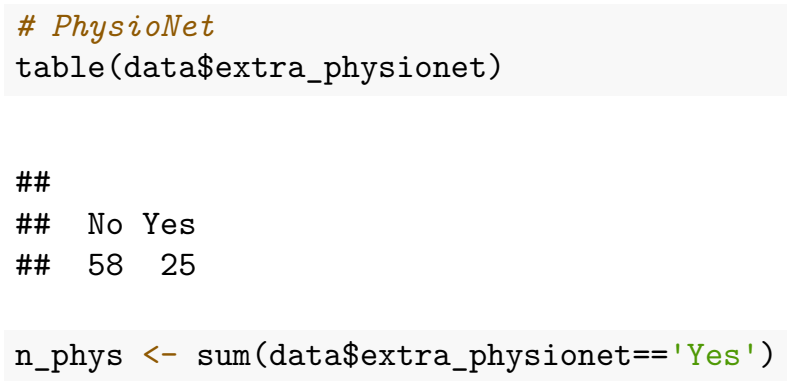

Two out of three articles utilized some open access dataset.

ImageNet-based models seemed to have a large impact on clinical studies even those analyzing non-image data.

The PhysioNet database was a common source for data with 25 studies utilizing data from this repository.

\section{Summaries by data types}

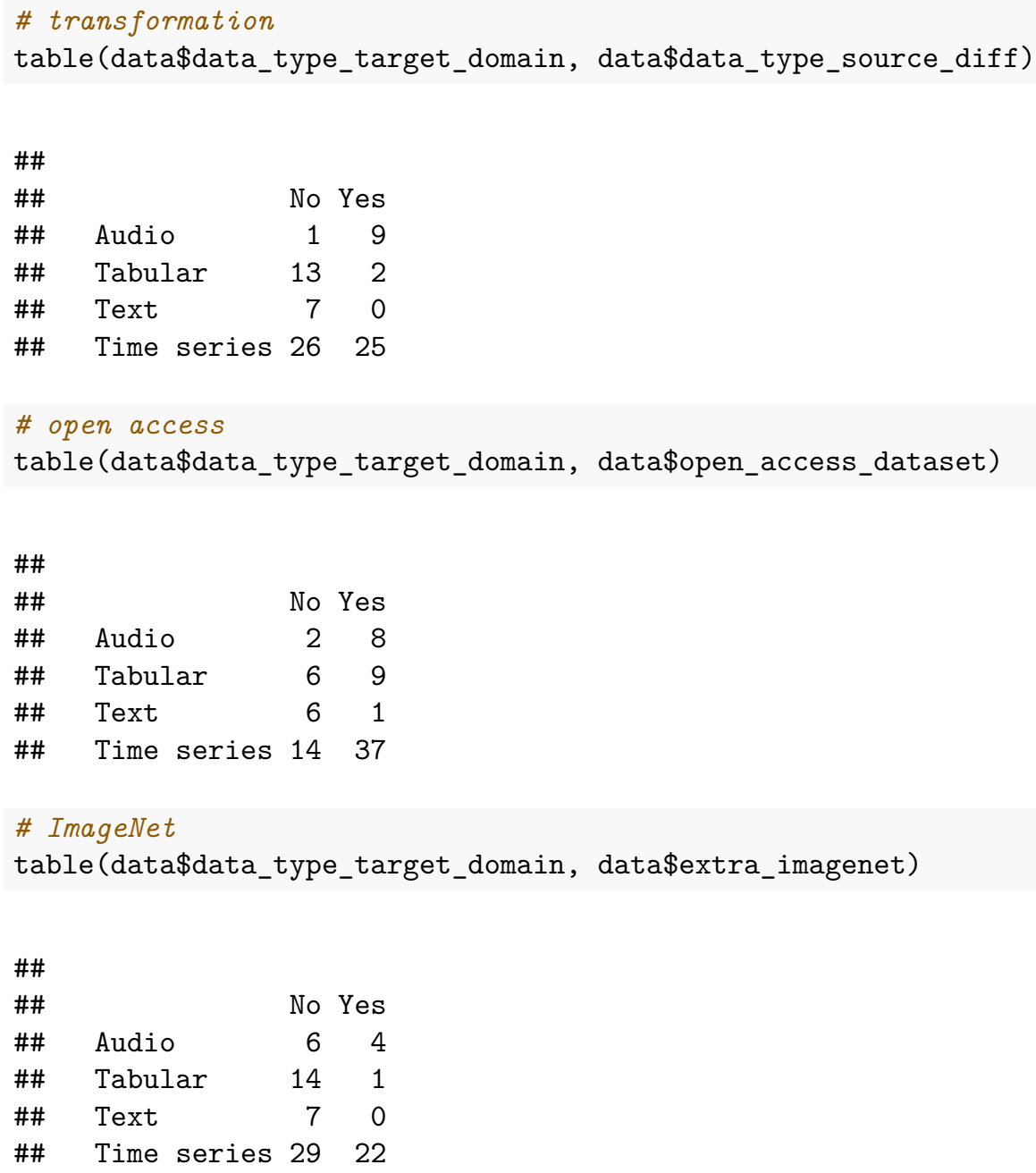


\# Physionet

table (data\$data_type_target_domain, data\$extra_physionet)

\#\#

\#\# $\quad$ No Yes

\#\# Audio $\quad 8 \quad 2$

\#\# Tabular $15 \quad 0$

\#\# $\quad$ Text 61

\#\# $\quad$ Time series 2922 